\title{
Terapia assistida por animais e transtornos do neurodesenvolvimento
}

\author{
Animal assisted therapy and neurodevelopmental disorders
}

Terapia asistida por animales y transtornos del neurodesarollo

\section{J éssica Riedi Souza Marinho*}

Centro Universitário FADERGS, Porto Alegre, Rio Grande do Sul, Brasil

Renata de Souza Zamo**

Centro Universitário FADERGS, Porto Alegre, Rio Grande do Sul, Brasil

\begin{abstract}
RESUMO
Este artigo visa levantar, por meio de construções teórico-científicas, os benefícios que a Terapia Assistida por Animais (TAA) pode gerar quando utilizada para tratamento com crianças que apresentam algum comprometimento neurodesenvolvimental. O contato humano-animal é conhecido desde a antiguidade, porém, apenas na atualidade estudos em torno deste viés estão crescendo. Portanto, foi realizada uma revisão crítica da literatura, analisando os dados coletados a partir de análise de conteúdo. Os resultados apontam o potencial benéfico deste tipo de intervenção com crianças com dificuldades sociais, cognitivas e físicas. Os animais mais utilizados são cachorros e cavalos para tratamento de crianças com Transtorno do Espectro Autista, Síndrome de Down e Deficiência Intelectual. Dessa forma, há uma possibilidade de que os profissionais da Psicologia, e de outras áreas da saúde, utilizem ou encaminhem para a Terapia Assistida por Animais (TAA) seus pacientes. Ainda assim, conclui-se que há a necessidade de mais estudos empíricos.
\end{abstract}

Palavras-chave: TAA, animais, crianças, transtornos, Psicologia.

\section{ABSTRACT}

This article aims to evaluate, through theoretical and scientific papers, the benefits that Animal Assisted Therapy (AAT) can provide to children who have some neurodevelopmental impairment. The benefits of human-animal contact has been known since antiquity, but studies around this area are starting to grow only currently. Therefore, we performed a critical review of the literature by analyzing the data collected using content analysis. The results showed the potential benefit of this type of intervention to children with social, cognitive and physical disabilities. The most commonly used animals were dogs and horses for treatment of children with Autism Spectrum Disorder, Down Syndrome and Intellectual Disabilities. Thus, there are possibility for psychologists and other professionals of health areas use or referee this kind of intervention to their patients. Still, we can conclude that there is a lack to more empirical studies using AAT.

Keywords: AAT, animals, children, disorders, Psychology. 


\begin{abstract}
RESUMEN
El presente artículo tiene por objeto evaluar, por medio de los aportes teóricos y científicos, los beneficios que la Terapia Asistida por Animales(TAA) puede generar cuando se la utiliza para el tratamiento de niños con algún tipo de compromiso en el desarrollo neurológico y mental. El contacto humano-animal es conocido desde la antigüedad, sin embargo, los estudios en torno a esta tendencia están empezando a crecer sólo en este momento. Por lo tanto, se realizó una revisión crítica de la literatura mediante el análisis de los datos obtenidos por medio del análisis de contenido. Los resultados muestran el beneficio potencial de este tipo de intervención con niños con dificultades sociales, cognitivas y físicas. Los animales más utilizados son el perro y el caballo para el tratamiento de los niños con trastorno del espectro autista, síndrome de Down y Discapacidad Intelectual. De este modo, existe la posibilidad de los profesionales de psicología, y otras áreas del salud, utilizar o indicar este medio de intervención para ayudar en el tratamiento de los pacientes. Aun así, se puede concluir que existe una necesidad de más estudios empíricos.
\end{abstract}

Palabras clave: TAA, animales, niños, transtornos, Psicología.

\title{
I ntrodução
}

A Psicologia na contemporaneidade busca inovar com diversos meios para promover a saúde mental, e a interação homem-animal é um destes. Este tipo de intervenção é chamado de Terapia Assistida por Animais (TAA) e conhecido oficialmente pelo mundo como Animal Assisted Therapy (AAT). A TAA pode ser realizada com qualquer pessoa, mas tem sido utilizada para incentivar o desenvolvimento biopsicossocial de pessoas com dificuldades físicas, emocionais, cognitivas e sociais (Capote \& Costa, 2011).

Os trabalhos já publicados mostram a inovação da TAA e a contribuição para essas pessoas e apontam principalmente a melhora na cognição, fala, socialização, autoestima, autocuidados, desenvolvimento físico, entre outros (Dotti, 2005). Estados Unidos, França e Canadá reconheceram cientificamente a Terapia Assistida por Animais, utilizando-a em diferentes áreas clínicas. Houve confusões terminológicas que necessitaram que a Delta Society (órgão responsável pela regulamentação de programas que utilizam animais nos Estados Unidos) determinasse uma nomenclatura oficial. Assim, diferenciaram termos como zooterapia, pet terapia, entre outros (Capote \& Costa, 2011). A Delta Society ainda dividiu a utilização animal em dois programas, a Atividade Assistida por Animais [AAA] e a Terapia Assistida por Animais [TAA]. O primeiro, caracterizado como um meio de recreação e entretenimento, sem a preocupação da realização de análise dos resultados nos pacientes. Por se tratar de uma terapia, a TAA é feita por profissionais específicos da área da saúde e busca o desenvolvimento e saúde do paciente (Capote \& Costa, 2011). 
No Brasil, essa função regulamentadora do uso animal como tratamento está em construção pela Rede Nacional de Atividade, Terapia e Educação Assistida por Animais [REATAA] (http://www.reataa.com.br/). Na constituição brasileira, há a Lei n. 11.794 (2008), que regulamenta o uso de animais como instrumentos de educação e pesquisa. Assim, cada instituição de ensino que os utiliza para estes fins, deve ter um Comitê de Ética no Uso de Animais [CEUA], garantindo o cumprimento da lei citada acima.

Problemas no desenvolvimento, sejam cognitivos, emocionais e/ou sociais, podem ser trabalhados com elevado índice de melhora quando logo identificados. A relevância da avaliação, para o adequado estabelecimento de metas de tratamento e as modalidades de intervenção neuropsicológica, auxiliam nos melhores resultados da reabilitação para transtornos do neurodesenvolvimento ou lesões encefálicas adquiridas (Ylvisaker et al., 2005). I sso se deve à capacidade de restauração do sistema nervoso quando ainda jovem e, portanto, o diagnóstico precoce e a melhor intervenção são aliados na promoção de saúde (Andersen, 2003; Brazelton, 1994; Muszkat, 2005).

Sobre a prevalência de transtornos mentais em crianças, segundo Cortello e Angold (1995) e Simonoff et al. (1997), como citado por Bee (2003), estima-se que varia de 14 a $20 \%$ a existência de alguma forma de psicopatologia em crianças e adolescentes por. Dentre essas, a deficiência intelectual é uma das mais frequentes. No censo demográfico de 2000 , foi identificado que $14,5 \%$ da população total brasileira apresenta alguma deficiência ou incapacidade (Capote, 2009), tendo prevalência de seis a cada 1000 pessoas, quando em casos graves, segundo a Associação Psiquiátrica Americana [APA] (2014). O DSM-5 (Manual diagnóstico e estatístico dos transtornos mentais), elaborado pela APA (2014) traz a prevalência de $1 \%$ na população, e estima-se que esse número seja parecido dentre adultos e crianças. É preciso levar em consideração que os estudos trazidos pela APA são com população norte-americana e de outras nacionalidades não mencionadas (APA, 2014).

No Brasil, Paula, Duarte e Bordini (2007) encontraram em uma amostra de um bairro de baixa renda paulistano a prevalência de $24.6 \%$ de crianças e adolescentes com algum transtorno mental de acordo com a classificação obtida no Child Behavior-Checklist e/ou Youth Self-Report (CBCL e YSR). Pode-se inferir que o início dos sintomas e o prognóstico de cada transtorno varia de acordo com os fatores de risco ou de oportunidades dos aspectos biológico, psicológico, social, familiar e cultural. Outro ponto que deve ser considerado é o momento vivenciado pela criança em seu estágio de desenvolvimento, quando ocorre algum comprometimento ou intercorrência. Assim, os mesmos fatores, bem como suas relações 
sociais podem gerar efeitos distintos para o funcionamento cerebral segundo a fase em que o indivíduo se encontra (Andersen, 2003; Dumas, 2011).

Beck e Katcher (1984) afirmam que intervenções com animais eram realizadas para proporcionar benefícios para os seres humanos, mas não tinham rigor metodológico e, portanto, sem comprovações científicas fidedignas. Hoje, com mais recursos, é possível obter maiores evidências de sua aplicabilidade em tratamentos. Por isso, trabalhos que agrupam os resultados de pesquisas empíricas são igualmente importantes para o crescimento da TAA.

Capote e Costa (2011) compilam pesquisas e trabalhos relacionados à utilização do animal para promover o bem-estar e melhoria do desenvolvimento de humanos. O vínculo idosos-animais vem sendo estudado desde o século passado. Em 1974, Mugford e M'Comisk (como citado em Capote \& Costa, 2011, p. 34-35), verificaram que a presença desses seres estava associada a um aumento na socialização das pessoas com seus familiares e amigos, ocasionando assim, um maior número de visitas em seus lares. Ainda, neste tipo de relação específica, Ory e Goldberg (1983) em estudo de comparação de grupos avaliaram a qualidade de vida de mulheres idosas que possuíam animais de estimação em comparação a mulheres idosas que não possuíam. O resultado obtido foi a correlação positiva entre felicidade e ter animais de estimação, portanto, quem tinha animais relatou ser mais feliz do que quem não tinha, essas se caracterizaram como infelizes.

Com pacientes cardíacos, verificou-se que donos de animais apresentaram maior sobrevida frente à doença em comparação com quem não tinha animal (Friedmann, Katcher, Lynch, \& Thomas, 1980). Além dos estudos de Edwards e Beck (2002), Sobo, Eng e Kassity-krich (2006) (citado em Capote \& Costa, 2011, p. 37-49) apresentam resultados de diversas pesquisas que relatam sentimentos de afeição despertados por esta interação em adolescentes, pessoas com necessidades especiais e pacientes nas seguintes situações: hospitalizados, com câncer, com demências, com dores, e com doenças terminais (Allen \& Blascovich, 1996, citado em Capote \& Costa, 2011, p. 37-49; Bjerke, Odegargstuen \& Kaltenborn, 1998, citado em Capote \& Costa, 2011, p. 37-49; Eddy, Hart \& Boltz, 1987, citado em Capote \& Costa, 2011, p. 37-49; Fraser, 1990, citado em Capote \& Costa, 2011, p. 37-49; Geisler, 2004, citado em Capote \& Costa, 2011, p. 37-49; Johnson et al., 2003, citado em Capote \& Costa, 2011, p. 37-49; Kanamori et al., 2001, citado em Capote \& Costa, 2011, p. 37-49; Lockwood, 1983, citado em Capote \& Costa, 2011, p. 37-49)

$\mathrm{O}$ autoconceito e o estresse também foram estudados por diversos pesquisadores como Allen, Blascovich e Mendes (2002), Allen et al. (1991), Poresky, (1997) e Raveis et al., (1993, citado em Capote \& 
Costa, 2011, p. 40-44). A partir destes e de outros estudos sugere-se que quando há o vínculo pessoa-animal, ter animais diminui o estresse e melhora o autoconceito (Poresky et al., 1988, citado em Capote \& Costa, 2011, p. 40-44; Siegel, 1990, citado em Capote \& Costa, 2011, p. 40-44).

Apesar do crescimento das TAAs, essa é pouco estudada nos cursos de graduação em Psicologia que tem a proposta de formar profissionais generalistas. Portanto, incentivar que os alunos aprofundem determinados temas de interesse e relevância como a TAA se torna fundamental. Além disso, é necessário ampliar a compreensão dos benefícios dessa terapia em crianças que apresentam alguma psicopatologia, auxiliando na possível utilização deste tipo de intervenção pelos profissionais da área. Realizar um levantamento bibliográfico de como esse método terapêutico é embasado teoricamente e empiricamente contribuirá na divulgação desta modalidade de tratamento.

Deste modo, este artigo tem por objetivo revisar na literatura as intervenções adequadas e satisfatórias da TAA no tratamento de crianças com comprometimentos neurodesenvolvimentais. Algumas questões norteadoras são apresentadas para contribuir no direcionamento do tema. Quais são os tipos de comprometimentos com maiores indicações do uso da TAA? Existem contraindicações referidas na literatura para a utilização desta terapia? E sobre os animais, quais são os mais utilizados e estudados? Pensando na contribuição da TAA para a Psicologia, existem abordagens teóricas específicas que a norteiam?

\section{Método}

Este estudo apresenta delineamento qualitativo, o que caracteriza a pesquisa em uma perspectiva mais indutiva, ou seja, seu enfoque se dá na interpretação da complexidade do tema trabalhado (Creswell, 2010). Assim, realizamos uma revisão da literatura sobre a Terapia Assistida por Animais e suas possíveis contribuições para o tratamento de crianças com transtornos mentais. Para isso, foi efetuado levantamento não sistemático de publicações científicas relacionadas ao tema.

Dentre os materiais selecionados por conveniência, há artigos científicos, dissertações e livros. Os artigos são provenientes das bases de dados a seguir: Scielo, Medline, PubMed e Google acadêmico. As buscas foram guiadas pelos seguintes descritores: Terapia Assistida por Animais, Pet Therapy, Animal Assisted Therapy, Equoterapia, bem como criança e child. Foram incluídas pesquisas publicadas entre os anos de 2002 a 2014. Foi realizada análise de conteúdo para compreensão dos dados. 
Segundo Bardin (1977), uma análise de conteúdo coerente, que objetiva a interpretação das mensagens que se deseja expor, deve ter como princípio uma organização. Esta é dividida em três etapas; na primeira, a pré-análise, consistiu na escolha dos documentos que foram utilizados e na formulação de hipóteses. No segundo momento, o material foi explorado e recortado agrupando em categorias de acordo com semelhanças e significados. Por fim, com o tratamento dos achados se fez a interpretação do material unido, buscando respostas para o tema investigado na elaboração da análise. Com a investigação realizada, foi possível dividir o conteúdo em três categorias, a Terapia Assistida por Animais (TAA), tipos de animais, que conta com as subcategorias o cachorro, o gato, o cavalo e outros animais e, por fim, a categoria abordagens teóricas.

\section{Resultados}

A partir da revisão dos artigos, dissertações e livros, foi possível verificar que os estudos abordam a TAA sob diferentes perspectivas. Esta revisão optou por selecionar estudos com crianças somente, assim os materiais com adultos e idosos auxiliaram na compreensão mais global da TAA. Para um maior aprofundamento apresentamos adiante as categorias trabalhadas.

\section{A terapia assistida por animais (TAA)}

Os materiais revisados apresentaram um apanhado histórico das Terapias Assistidas por Animais. A interação entre humanos e animais é conhecida há milhares de anos e reconhecida até hoje (Garcia, 2005). A relação terapêutica dessa junção também é antiga, ao contrário do que muitos podem pensar. Documentos da antiguidade romana já apontavam o poder de cura de cães, considerados sagrados (Levinson, 1965). Em 1973, Ainsworth (como citado em Capote \& Costa, 2011, p. 33), ao estudar o vínculo entre homemanimal, compara-o ao vínculo mãe-bebê, porque a relação emocional oriunda dessas interações invadem espaço e tempo, podendo interferir no desenvolvimento humano. No Brasil, os primeiros trabalhos registrados que utilizaram animais foram realizados pela Dra. Nise da Silveira, na década de 1950. Já internacionalmente, o precursor da Terapia Assistida por Animais foi Boris Levinson, em 1962 (Dotti, 2005). Com estes marcos históricos, pode-se analisar que o uso da TAA foi iniciado no Brasil antes do quadro internacional encontrado por esta pesquisa Porém, o reconhecimento da técnica terapêutica ainda é muito tardio nesse país.

Depois da década de 1990, as pesquisas sobre o tema começaram a apresentar rigor metodológico e cientificidade, auxiliando na 
compreensão sobre a TAA (Capote \& Costa, 2011). Nas pesquisas já existentes, há a possibilidade de dividí-las nas que analisam o envolvimento natural com donos e seus animais e as que fazem da utilização da relação animal/pessoa uma forma de intervenção. Porém, as maneiras de intervenção e as formas de condução não deixam claras as possíveis vantagens e desvantagens desta terapia (Siegel, 1993, como citado em Capote \& Costa, 2011, p. 28). Reportagens midiáticas apontam os benefícios desta interação entre espécies, embasadas principalmente em pesquisas internacionais e, também, em programas de atendimentos que foram realizados no Brasil, na maioria, executados em instituições que atendem idosos e crianças, hospitais, abrigos e universidades (Capote \& Costa, 2011). Outro fator relevante são as contraindicações na TAA, Na literatura há mais referências de que os animais utilizados em terapia devem estar em boas condições de saúde para que a interação não prejudique o paciente, ou seja, sem presença de pulgas, carrapatos e zoonoses, vacinações em dia, limpos, escovados e com higienização anterior e posterior ao contato com o paciente. O acompanhamento com veterinário dos animais é um tema referido como primordial (Owen, 2002 como citado em Pereira, Pereira \& Ferreira, 2007, p. 64).

Lamentavelmente, não há muitos dados sobre quais são os pacientes que melhor respondem a esta intervenção, embora tenhamos escritos sobre para quais pacientes devemos contraindicar a TAA. Devido à complexidade do ser humano, a TAA possui algumas contraindicações, como nos casos de alergias, pacientes com problemas respiratórios, fobias de animais, pacientes com machucados abertos, pacientes com baixa imunidade, além dos que apresentam comportamentos agressivos que podem machucar 0 animal (Stanley, 2002, como citado em Pereira, Pereira \& Ferreira, 2007 , p. 64). O local onde se realiza a intervenção também deve ser rigidamente controlado e inspecionado para verificar a existência de possíveis agentes infectantes (Connor, 2000, como citado em Pereira, Pereira \& Ferreira, 2007, p. 64).

A Terapia Assistida por Animais aborda cinco mecanismos de ação conforme a categorização de Rose, Cannas e Cantielo (2011) que são: a) afetivo-relacional, enfatiza a força do vínculo humano-animal; b) estímulo psicológico, o vínculo estabelecido age na psique humana, proporcionando melhoras de comportamento sóciorelacional, caráter e cognitivo; c) recreacional, cujas brincadeiras estimulam a autoestima, diminuem o isolamento social, e geram mudanças positivas no humor; d) psicossomático, e; e) físico. Estes últimos representam a ação principal sobre o corpo do indivíduo com relação aos três primeiros. 
Tipos de animais

Esta categoria foi dividida em subcategorias pois há características específicas de cada animal que consideramos relevante pontuar. Ainda assim, esta variabilidade não permite muitos parâmetros de comparação devido à heterogeneidade tanto de animais quanto de grupos clínicos. Logo, a apresentação dos estudos fica mais clara quando em categorias distintas.

\section{O cachorro}

Segundo Anderlini (2009, como citado em Fulber, 2011), o cão, e o convívio com ele, traz diversos benefícios emocionais para os seres humanos, independentemente de classe social, condições de saúde e faixa etária das pessoas. É referido que este proporciona observáveis melhoras na qualidade de vida. A maioria das pesquisas que investigam os benefícios e impactos da TAA é realizada com cachorros. Fato que facilita o entendimento dos resultados gerados pela intervenção quando há o uso do cão.

Os estudos realizados com cachorros identificaram benefícios significativos em crianças com paralisia cerebral nas variáveis autonomia, integração social e habilidades motoras, que obtiveram aumento durante as avaliações periódicas (Fosco et al., 2009; Poutechi \& Coutinho, 2011). Os cães comunmente são utilizados em pesquisas e tratamentos em centros de saúde, como aconteceu na Associação para Valorização de Pessoas com Deficiência [AVAPE], que aplicou a TAA em crianças com deficiência intelectual, no intuito de saber se haveria melhoras na socialização destas após a terapia. O resultado quanto à receptividade e ao contato interpessoal antes da intervenção era de $65 \%$ e depois foi de $90 \%$. Neste estudo, a TAA foi realizada em 12 encontros, obtendo maiores resultados a partir da 8a sessão. Referente ao relacionamento, houve melhora de 35\% dentre os participantes que obtiveram maiores pontuações nas avaliações feitas antes e após o tratamento. Esta pesquisa ainda verificou melhora na organização do pensamento e da sequência dos acontecimentos que foram avaliados a partir da análise de narrativas da experiência da TAA (Vivaldini, 2011).

O cachorro, ao ser introduzido na relação paciente-terapeuta faz o papel de mediador, auxiliando como fonte de comunicação entre as partes (Vivaldini, 2011). Quando em contato com os seres humanos, os cachorros promovem melhora na socialização e na saúde mental (Cadth, 2012). Por serem animais de grande expressão de afetividade, pode-se pensar na possibilidade de, quando em interação com crianças, promoverem significativas respostas emocionais, ajudando no desenvovimento afetivo. O autocuidado e a autoestima também podem ser estimuladas pela necessidade do cuidado que 
eles devem ter com aquele ser (cachorro). Althausen (2006), Rose et al. (2011) e outros estudos referem melhora na comunicação, o que favorece crianças com dificuldades verbais devido à psicopatologia (Amorim et al., 2004, citado em Capote \& Costa, 2011, p. 58; Melson, 1990, citado em Capote \& Costa, 2011, p. 50).

O gato

Os gatos são reverenciados pelos seres humanos há muito tempo, sendo objeto de estudos frente a suas simbologias. Dentre a espécie felina, os felis catus, mais conhecidos como gatos domésticos, são, juntamente com os cães, os animais mais domesticados pelos seres humanos (http://pt.wikipedia.org/wiki/Gato). Isto se dá pela admiração que o homem tem pelo caráter e temperamento desses animais. Devido a este fato, eles vêm sendo usados em tratamentos com pessoas que apresentam dificuldades psíquicas (Dotti, 2005).

Porém, Dotti (2005) salienta que os gatos possuem uma personalidade mais independente, são orgulhosos e podem não aceitar nossos caprichos humanos. Algumas pessoas podem se identificar, fazendo com que o tratamento seja mais positivo, mas, para outras, pode ser um empecílho, principalmente aqueles que esperam do gato uma postura mais sociável. A sociabilidade desta espécie acontece da maneira que o animal deseja, quando the convém. Além disso, alguns pacientes mais sensíveis não podem ter contato com este tipo de animal devido a complicações de saúde como feridas, alergias e pele sensível. Talvez, por estes motivos, os médicos acabam indicando um maior uso terapêutico do cachorro, ao invés do gato.

Dentre as pesquisas revisadas, poucos materiais abordavam 0 trabalho terapêutico com gatos. Destes, nenhum se referia a tratamento com crianças. Este fenômeno pode ser pensado por causa da instabilidade do temperamento felino e pelos riscos de saúde elevados quando comparados a outros tipos de animais. Entretanto, Dotti (2005) cita o estudo do Doutor Dennis Turner que refere mudanças comportamentais positivas em pessoas que se sentiam mais deprimidas e menos ativas após se relacionarem com gatos. A Dra. Nise da Silveira, pioneira em intervenções com animais no Brasil, utilizou gatos para promover qualidade de vida de pacientes esquizofrênicos de um hospital psiquiátrico (Pereira, Pereira, \& Ferreira, 2007).

O cavalo

O cavalo, símbolo de soberania, altivez, trabalho, paixão e, principalmente, força, vem contribuindo muito para o homem durante os anos de interação. Assim sendo, Dotti (2005) orienta mais 
analiticamente sobre o poder desse animal na vida de um ser humano:

Refletimos no cavalo nossos desejos de sermos fortes e livres. A experiência de montar em um cavalo e trabalhar com ele nos leva ao sentimento de que podemos "Ter" o controle deles e isso, inscientemente, nos revela o controle sobre nossa própria força. O cavalo nos aproxima de nossas fraquezas, transformando-as. Ajuda-nos a enfrentar nossas sombras e nossos medos, pois encontramos nele aquilo que realmente queremos ser (p. 27).

A Terapia Assistida por Animais quando realizada com o cavalo é denominada, no Brasil, de Equoterapia. A utilização deste animal é variavelmente diferenciada entre algumas terapias, quando citadas internacionalmente, pois existem diversas nomenclaturas, como "Therapeutic Horseback Rinding", "Hippotherapy" e "Equine-Assisted Psychotherapy". Apesar delas usarem o cavalo como meio terapêutico, a primeira teria seu foco mais abrangentemente, trabalhando sob um viés psicológico, social, físico e educacional. A segunda, focaria mais nos fatores da motricidade, postura e equilíbrio. Por fim, a terceira é vista quando o próprio terapeuta é também o instrutor e a figura do animal é considerada terapêutica basicamente pela sua presença (Holm et al., 2014). Dentre os tantos nomes para estes tipos de tratamentos com o cavalo, Leitão (2004, 2008) sugere um agrupamento desses e mais alguns (Equitação desportiva e também Equitação psico-educacional), citando a Equitação terapêutica como uma intervenção tridimensional, onde todos os focos são englobados. Muitos dos estudos (Ajzenman, Standeven \& Shurtleff, 2013; Gabriels et al., 2012; Hameury et al., 2010; Holm et al., 2014; Keino et al., 2009; Lanning et al., 2014; Memisevikj \& Hodzhikj, 2010) encontrados durante esta pesquisa eram direcionados para crianças com transtornos globais do desenvolvimento, principalmente o autismo. Atualmente, classificados como transtornos do neurodesenvolvimento, segundo o DSM-5 (APA, 2014).

O estudo de Keino et al. (2009) avaliou três crianças com TEA e duas com transtorno do desenvolvimento pervasivo com a escala HEIM (Human-Equips-Interaction on Mental activity). Esta avalia a interação entre o indivíduo e a equipe em 10 itens comportamentais: 1) Relações humanas; 2) Imitações; 3) Expressar emoções; 4) Movimentos físicos; 5) Comportamentos fixos; 6) Adaptações a mudanças; 7) Resposta visual; 8) Medo ou nervosismo; 9) Comunicação verbal, e; 10) Comunicação não verbal. Foram encontrados resultados significativos na maioria dos itens, exceto em 
movimentos físicos, comportamentos físicos e comunicação não verbal.

Outro trabalho com crianças com TEA foi uma comparação entre tratamento equoterápico e um programa de intervenção social. Porém, neste estudo, houve uma variável interveniente que impediu a comparação dos dados. Alguns participantes não preencheram os questionários, sendo excluídos da análise. Entretanto, a pesquisa que foi conduzida com outros métodos de avaliação auxiliaram na obtenção de dados. Uma hipótese confirmada foi a melhora na qualidade de vida em ambos os grupos. A segunda hipótese foi a possibilidade de o grupo com tratamento equino ter maior qualidade de vida quando comparado com o grupo intervenção social. Os resultados apontaram para uma significância apenas na funcionalidade física e psicossocial no grupo com Equoterapia (Lanning et al., 2014).

Quando se refere à hipoterapia, Ajzenman et al. (2013) elaboraram um estudo piloto com crianças com TEA para testar a hipótese de que a terapia aumentaria os comportamentos adaptativos, melhoraria as atividades de vida diárias e o controle motor dos participantes. Os dados encontrados sugerem respostas positivas quanto aos constructos avaliados sugerindo em um aumento de habilidades sociais.

A equitação psico-educacional corroborra com a perspectiva das melhorias que o auxílio terapêutico do cavalo pode proporcionar. Leitão (2003) sugere em seu estudo que a criança com TEA geralmente possui uma diferenciação entre a idade desenvolvimental e a idade cronológica. Este tipo de intervenção promove aumento do desenvolvimento global destas crianças, reduzindo a distância entre estas idades.

O tratamento com a utilização do cavalo também evidenciou melhoras significativas em crianças com Síndrome de Down. Como fatores positivos, o estudo apontou maior interação social, independência emocional e física, autocuidado, e também benefícios na motricidade (Campos, 2007).

É perceptível que a terapia com foco biopsicossocial através da interação com o cavalo já é estudada e com comprovações científicas positivas, quando comparada com estudos de mediação com outro tipo de animal, como, por exemplo, gatos, pássaros e outros. Isso pode se dar devido a interação homem-cavalo ser mais convencional, pela possibilidade de realizar a montaria, e pela grande adaptabilidade, possivelmente pela maior acessibilidade de vínculo, na medida em que outros animais tendem a ser ariscos e de menor facilidade de adestramento. 


\section{Outros Animais}

Outros animais podem ser usados em intervenções com crianças com transtornos mentais, como realizado em um CAPS-I [Centro de Atenção Psicossocial - Infantil] na cidade de Porto Alegre. Os participantes eram dois grupos de crianças e adolescentes atendidos na instituição, portadores de diversos transtornos mentais, como: transtorno de déficit de atenção e hiperatividade (TDAH), transtorno de conduta, transtorno de humor, transtorno opositor desafiante e vítimas de abuso sexual. Os animais utilizados foram tartaruga, cachorro, hamster, peixe, esquilo chinês, coelho, cabra, porco da índia e periquito. O grupo A era de participantes com menor independência, mais jovens, e o grupo B de idade superior e de maior independência. A avaliação antes e após a intervenção foi com a Children Global Assessment Scale (CGAS) e o Questionário de Capacidades e Dificuldades (SDQ) versão para pais. Os resultados dos jovens foram significativos e indicaram melhora nas dificuldades enfrentadas após a TAA. Porém, as respostas dos responsáveis não mostraram diferenças significativas após o tratamento (Faraco et al., 2009).

Os autores defendem a intervenção pela relevância dada aos seres vivos, para explorar uma relação completamente diferente. O contato com os animais estimulou as crianças e os adolescentes a usar novas palavras, medir comportamentos e fazeres, o que os motivou a identificar, alterar (ou não) atitudes e comportamentos, a partir desta nova experiência. Portanto, Faraco et al. (2009) concluiram que esta mediação, como tratamento complementar, traz benefícios para os que a utilizam.

Um estudo piloto realizado na Itália, no Child Neuropsychiatry Outpatient Unit do Istituto S. Giovanni di Dio Fatebenfratelli, diversificou usando um burro. Este animal é um facilitador na construção de motivação, estimulando o crescimento da criança a partir de forças positivas e ativas necessárias no processo desenvolvimental psicocognitivo e psicoafetivo. Os participantes tinham entre seis e 12 anos, com pelo menos uma das seguintes condições: distúrbio em relações emocionais, distúrbios comportamentais, deficiência intelectual, dificuldades de comunicação, hiperatividade e/ou sintomas de depressão e sofrimento psíquico. O estudo demonstrou que para os participantes com dificuldades de linguagem, embora a linguagem usada com o animal seja mais corporal, a interação apresentou efeitos terapêuticos benéficos na comunicação. Os desenhos dos participantes antes e depois das sessões sugeriram uma conquista de percepção de sua representação pessoal e com o mundo exterior mais desenvolvida após a intervenção. Segundo os autores, o grupo estava mais capacitado a expressar suas relações, vontades e sua 
própria personalidade. A pesquisa auxiliou a equipe terapêutica a ter maior compreensão sobre os sentimentos das crianças, auxiliando no potencial das atividades de reabilitação por apresentar pontos fortes destas indicando o que pode ser investido para promover a motivação deles no tratamento (Rose et al., 2011).

Programas terapêuticos com golfinhos são conhecidos mundialmente. Uma pesquisa exploratória investigou a interação de crianças com TEA e golfinhos durante 12 semanas. Alguns aspectos avaliados não foram significativos, como reatividade emocional, comunicação e orientação social, quesitos comportamentais, exploração perceptosensorial, imitação, coordenação olho-mão e motricidade global. Ainda assim, a comunicação não verbal apresentou melhora importante. Após 11 meses do término da intervenção, foram observadas manutenção de benefícios em desenvolvimento global, motricidade fina, desempenho cognitivo e cognição verbal. Os comportamentos dos participantes eram impressionantes perante os pais quando em contato com o animal, e eles diziam perceber diferenças fora dali, o que motivou a continuidade do programa terapêutico. Os autores citaram duas publicações em que houve aumento significativo de interação, mostrando que há incremento no interesse, envolvimento e aceitação da família para as crianças com dificuldades mentais quando nesse tratamento (Salgueiro et al., 2012).

A TAA com animais não domésticos ainda é pouco estudada, o que dificulta o entendimento de possíveis benefícios para a saúde mental infantil. Porém, com os dados existentes, é visível a importância no desenvolvimento de alguns aspectos na vida de crianças que possuem algum comprometimento biopsicossocial.

\section{Abordagens Teóricas}

Althausen (2006) traz resultados qualitativos dos encontros de adolescentes com Síndrome de Down e cães, analisando-os à luz da psicanálise winnicottiana, como a expressão dos sentimentos e dos aspectos do self de cada participante. A autora destaca que essa liberação seria o efeito da interação e que cada cão despertava um tipo de aspecto do self em cada um dos participantes. Sentimentos que eles consideravam difíceis de demonstrar com outros humanos emergiam na interação com os animais.

Outra publicação psicanalítica relata o caso de uma adolescente atendida com cães domésticos. Além dos cães, hamsters também foram utilizados em busca de melhores resultados. Com a mediação, principalmente dos cães, a psicanalista relatou ter conseguido estabelecer uma boa relação com a paciente, desenvolvendo transferência e elucidando os mecanismos de defesa da garota em suas falas e comportamentos (Minerbo, 2002). Pode-se compreender 
que o animal seja um elemento a mais a serviço da projeção e da transferência.

A teoria do apego vêm sendo discutida por alguns autores quanto à utilização desta em intervenções com animais, como relata Bachi (2013) em estudos de revisão com cavalos. Porém, há contradições. Enquanto Philips Cohen (2007 como citado em Bachi, 2013, p. 188) diz que a teoria do apego pode auxiliar no tratamento com animais, pelo surgimento de insights quando pensado a partir do que envolve as relações interpessoais, Endenburg (1995 como citado em Bachi, 2013, p. 188) diz que o apego entre humanos e animais é diferente, que estaria localizado em partes distintas do funcionamento interno. Corroborando com o último, Zilcha-Mano et al. (2011, como citado em Bachi, 2013, p. 188-189) contempla que as orientações de apego entre seres humanos e humanos-animais possuem uma estreita relação, mas podem ser relatados separadamente.

Ainda assim, Bachi (2013) buscou na teoria do apego de J. Bowlby o que poderia ser produtivo na Equoterapia. Uma de suas conclusões é que a ausência de um método que avalie e comprove a eficácia da junção de ambas intervenções prejudica uma maior cientificidade deste aspecto. Bachi (2013) salienta que as contribuições iniciais da teoria do apego correspondem a algumas das principais características geradas também pela terapia com cavalos, como uma "base segura e refúgio através do fornecimento de um ambiente de exploração seguro, usa o espelhamento, a mentalização e funcionamento reflexivo, comunicação não-verbal e experiência do corpo" (p. 190).

Embora os estudos supracitados citem seus referenciais teóricos, havendo referência somente na abordagem psicanalítica, muitas das publicações encontradas não faziam referências quanto às abordagens em Psicologia. Isso pode acontecer devido aos estudos serem exploratórios e pilotos ou revisões da literatura, indicando carências de sustentação teórica e de coleta de resultados empíricos nas pesquisas a fim de argumentar a validade da intervenção com consistência.

\section{Considerações finais}

Pensando a partir do desenvolvimento dos mecanismos relacionais e de comportamentos sociais, os animais podem vir a desempenhar um papel de estimulantes sociais, encorajando a comunicação (Rose et al., 2011). Além disso, os estudos mostraram que tal relação auxilia o desenvolvimento da comunicação verbal, não verbal e corporal.

Ao pensar sobre o auxílio do animal como mediador de um processo de tratamento psicológico, é viável compará-lo às atividades lúdicas, devido à utilização de simbolismos e comunicação não-verbal. Nessas 
brincadeiras e brinquedos (assim como pode ocorrer com os animais), a criança coloca seus sentimentos, que representam suas relações internalizadas (Efron et al., 1978, como citado em Cunha, 2007, p. 99). É possível observar diversos fenômenos em que a criança projeta questões do seu mundo interno e os pontos-chave a serem trabalhados (Logan, 1991, como citado em Cunha, 2007, p. 99). De acordo com Cunha (2007), o psicólogo deve manter uma postura que venha a estimular a interação, agindo de modo que venha a conduzir o processo de tal forma que deixe a compreensão do momento exposta, acolhendo e demonstrando respeito, para a criança se sentir segura e aceita naquele ambiente.

Segundo a revisão realizada, o crescimento afetivo, emocional e o processo de construção de caráter pode ser promovido através da interação homem-animal pela troca afetiva e a gratificação que é estimulada a partir do reforço positivo dado pelo animal. Esses fatores coincidem para melhorar a autoimagem e autoestima. Ainda, contribuem para o senso de responsabilidade, sendo também um meio de liberação das emoções. Quanto à cognição, o contato com animais promove nas crianças foco e curiosidade, motivando-as a buscar entendimento sobre animais, diversidade, natureza, e consequentemente, aprendizado geral (Rose et al., 2011). É importante salientar que, devido ao fato de ser uma modalidade de tratamento de exploração inicial, a utilização da TAA deve ser concomitante a outros tipos de tratamentos terapêuticos, conforme cita Faracco et al. (2009).

Com os dados levantados neste artigo, pode-se concluir que a TAA traz benefícios significativos para a vida de qualquer indivíduo, e, possivelmente, independe do tipo de animal, como mostrado por Faraco et al., 2009 (desde que haja um treinamento e o mediador tenha controle do animal). Este tipo de intervenção ajuda a promover melhoras cognitivas, físicas, emocionais e sociais. Apesar disso, a diversificação de animais em estudos publicados é baixa, pois parte destes foram realizados com cachorros e cavalos, os mais comuns, e possivelmente os mais fáceis de serem treinados frente a comportamentos inesperados das crianças (Ajzenman et al., 2013; Althausen, 2006; Campos, 2007; Capote, 2009; Fosco et al., 2009; Holm et al., 2014; Lanning et al., 2014; Leitão, 2004; Poutechi \& Coutinho, 2011).

Segundo Vaccari e Almeida (2007), os vínculos com animais são mais indicados para crianças, devido ao animal se consagrar como uma fonte de inspiração e admiração para elas, justamente pela busca de conquistas de seus objetivos e pela idealização de realizações. E, acima disso, a relação entre criança-animal é entrelaçada forte e afetivamente, gerando responsabilidade e afeição de ambas as partes. A TAA é considerada de grande auxílio para melhorar a situação de saúde de crianças hospitalizadas. 
Houve limitações de resultados mais recentes e com crianças que possuem outros diagnósticos que não seja TEA, o que surgiu com frequência, principalmente nas pesquisas de Equoterapia. Assim, é difícil pensar tanto qual animal, quanto qual transtorno é o mais adequado. Analisando os resultados apresentados, é observável que as crianças com Transtorno do Espectro Autista e Síndrome de Down podem ser beneficiadas pelos cavalos e cachorros. A deficiência intelectual é apresentada com maiores resultados quando realizada com cães. Para se ampliar o conhecimento sobre outras psicopatologias e suas associações com os animais, no foco de tratamentos psicológicos, é necessário mais estudos sobre a atuação do profissionais da Psicologia. Por ser uma técnica nova, estes estudos poderiam auxiliar psicólogos que têm interesse em utilizá-la ou a encaminharem e idicarem este tipo de tratamento. A ampliação de estudos também poderia fomentar a abertura de centros de atendimento em TAA e contribuir para o atendimento e estudo com outros grupos de pessoas com comprometimentos psicológicos.

No que se refere às contraindicações, variam muito, pois é necessário levar em consideração os aspectos do animal e características do paciente. Praticamente nenhuma publicação revisada explicitou alguma limitação seja psicopatológica, seja de outros comprometimentos infantis. Apenas as contraindicações dos animais são pontos a serem observados primordialmente. Por outro lado, aqueles que trabalham com Equoterapia realizam uma avaliação prévia seguindo algumas contraindicações referidas pela Associação Nacional de Equoterapia nos cursos de habilitação em Equoterapia (ANDE/BRASI L, 2001).

Há a necessidade de mais estudos empíricos com outros tipos de comprometimentos neurodesenvolvimentais, de psicopatologias infantis para comprovar cientificamente a TAA. Além disso, pesquisas com outros animais menos utilizados para domesticação e comprovações de abordagens teóricas para o embasamento das técnicas dos profissionais precisam ser elaboradas. Dessa forma, provavelmente, teremos programas mais definidos e conhecimentos de sua eficácia para a aplicação da intervenção psicológica com animais.

\section{Referências}

Ajzenman, H. F., Standeven, J. W., \& Shurtleff, T. L. (2013). Effect of hippotherapy on motor control, adaptative behaviors, and participation in children with autism spectrum disorder: a pilot study. The American Journal of Ocupation Therapy, 67(6), p. 653-663. 
Allen, K., Blascovich, J., \& Mendes, W. B. (2002). Cardiovascular reactivity and the presence of pets, friends, and spouses: the truth about cats and dogs. Psychosomatic Medicine, 64, p. 727739. Recuperado em 27 janeiro, 2017, de http:// wendyberrymendes.com/cms/uploads/Allen2002$\%$ 20Cardiovascular\% 20reac. pdf

Allen, K., Blascovich, J., Tomaka, J., \& Kelsey, R. M. (1991). Presence of human friends and pet dogs as moderators of autonomic responses to stress in women. Journal of Personality and Social Psychology, 61(4), p. 582-589. Recuperado em 27 janeiro, 2017, de https://www.calvin.edu/ rpruim/courses/m143/articles/Petstre ssArticle.pdf

Althausen, S. (2006). Adolescentes com síndrome de down e cães: compreensão e possibilidades de intervenção. (Dissertação de mestrado não publicada). Universidade de São Paulo, São Paulo, SP, Brasil. Recuperado em 20 abril, 2015, de http://www.teses.usp.br/teses/disponiveis/47/47131/tde13092006-154744/publico/ALTHAUSEN.pdf

American Psychiatric Association. (2014). Manual diagnóstico e estatístico de transtornos mentais, DSM-5 ( 5 a ed.). (M. I. C. Nascimento et al., Trad.). Porto Alegre: Artmed.

ANDE/BRASIL (Associação Nacional de Equoterapia - ANDE-BRASIL). (2001). Coordenação de Ensino e Pesquisa e Extensão - COEPE (Eds.), Polígrafo do $40^{\circ}$ Curso Básico de Equoterapia. Brasília, Dez.

Andersen, S. (2003). Trajectories of brain development: point of vulnerability or window of opportunity?. Neuroscience and Behavioral Reviews, 27, 3-18.

Bachi, K. (2013). Application of attachment theory to equinefacilitated psychotherapy, Journal Contemp. Psychotherapy, 43, 187-196.

Bardin, L. (1977). Análise de Conteúdo. Lisboa: Edições 70.

Beck, A. M., \& Katcher, A. H. (1984). A new look at pet-facilitated therapy, JAVMA, 184(4), p. 414-423.

Bee, H. (2003). A criança em desenvolvimento (9a ed.). (M. A. V. Veronese, Trad.). Porto Alegre: Artmed.

Brazelton, T. B. (1994). Momentos decisivos no desenvolvimento infantil. São Paulo: Martins Fontes.

Campos, C. S. (2007). Equoterapia - O enfoque psicoterapêutico com crianças down. Monografia. Pontíficia Universidade Católica de Goiás, Goiania, GO, Brasil. Recuperado em 18 abril, 2015, de http://equoterapia.org.br/site/files/trabalhos/24102031.pdf

Canadian Agency for Drugs and Technologies in Helth [CADTH] (2012). Therapy dogs and horses for mental health: a review of the clinical effectiveness. Recuperado em 06 abril, 2015, de 
https: // www.cadth.ca/sites/default/files/pdf/htis/aug2012/RC0381\% 20Therapy\% 20Animals\% 20final. pdf

Capote, P. S. O. (2009). Terapia Assistida por Animais (TAA) e deficiência mental: análise do desenvolvimento motor. (Dissertação de mestrado não publicada). Universidade Federal de São Carlos, São Carlos, SP, Brasil. Recuperado em 03 novembro, 2014, de http: //livros01.livrosgratis.com.br/cp144972.pdf

Capote, P. S. O., \& Costa, M. P. R. (2011). Terapia Assistida por Animais (TAA): aplicação no desenvolvimento psicomotor da criança com deficiência intelectual. São Carlos, SP: Editora UFSCar.

Creswell, J. W. (2010). Projeto de pesquisa: métodos qualitativo, quantitativo e misto ( $3 a$ ed.). Porto Alegre: Artmed.

Cunha, J. A. (2007). Psicodiagnóstico-V. Porto Alegre: Artmed.

Dotti, J. (2005). Terapia e animais. São Paulo: Noética.

Dumas, J. E. (2011). Psicopatologia da infância e da adolescência. Porto Alegre: Artmed.

Edwards, N. E., \& Beck, A. M. (2002). Animal-assisted therapy and nutrition in Alzheimer's Disease, Western Journal of Nursing Research, 24(6), 697-712. Recuperado em 27 janeiro, 2017, de https: // habricentral.org/resources/460/download/WJ NR_Edwar dsBeck_FishAlzheimers.pdf

Faraco, C. B., Pizzinato, A., Csordas, M. C., Moreira, M. C., Zavaschi, M. L. S., Santos, T., ... Menti, L. M. (2009). Terapia mediada por animais e saúde mental: um programa no centro de atenção psicossocial da infância e adolescência em Porto Alegre - TAA parte III, Saúde Coletiva, 6(34), p. 231-236.

Fosco, M. M., Ribeiro, P. R., Ferraz, F. H. A., Freitas, R., Jr., Martin, D. W., Raymundo, C. S., \& Pereira, C. A. D. (2009). Aplicação da terapia assistida (TAA) por animais no tratamento de crianças portadoras de paralisia cerebral - TAA - Parte I, Saúde Coletiva, 32(6), p. 174-180.

Friedmann, E., Katcher, A. H., Lynch, J. J., \& Thomas, S. A. (1980). Animal companions and one-year survival patients after discharge from a coronary care unit, Public Health Reports, 95(4), 307-312. Recuperado em 27 janeiro, 2017, de https://www.ncbi.nlm.nih.gov/pmc/articles/PMC1422527/pdf/p ubhealthrep00128-0003.pdf

Fulber, S. (2011). Atividade e terapia assistida por animais. Trabalho de conclusão de curso, Universidade Federal do rio Grande do Sul, Porto Alegre, RS, Brasil. Recuperado em 02 abril, 2015, de https: //www. lume.ufrgs. br/bitstream/handle/10183/52516/000 851221.pdf? sequence $=1$

Gabriels, R., Agnew, J., Holt, K., Shoffner, A., Zhaoxing, P., Ruzzano, S. ... Mesibov, G. (2012). Pilot study mensuring the effects of 
therapeutic horseback riding on school-age children and adolescents with autism spectrum disorders. Research in Autism Spectrum Disorders, 6, 578-588.

Garcia, R. C. (2005). Controle de populações de cães e gatos em área urbana: uma experiência inovadora na Grande São Paulo, Saúde Coletiva, 2(5), 24-28.

Hameury, L., Delavous, P., Teste, B., Leroy, C., Gaboriau, J.-C., \& Berthier, A. (2010). Équithérapie et autisme. Annales MédicoPsychologiques, 168, 655-659.

Holm, M. B., Baird, J. M., Kim, Y. J., Rajora, K. B., D'Silva, D., Podolinsky, ... Minschew, N. (2014). Therapeutic horseback riding outcomes of parent-identified goals for children with autism spectrum disorder: an $\mathrm{ABA}^{\prime}$ multiple case design examining dosing and generalization to the home and community, Journal of Autism Developmental Disorder, 44, p. 937-947.

Keino, H., Funahashi, A., Keino, H., Miwa, C., Hosokawa, M., Hayashi, Y., \& Kawakita, K. (2009). Psycho-educational horseback riding to facilitate communication ability os children with pervasive developmental disorders, Journal Equine Science, 20(4), 79-88.

Lanning, B. A., Baier, M. E. M., Ivey-Hatz, J., Krenek, N., \& Tubbs, J. D. (2014). Effects of equine assisted activities on autism spectrum disorder, Journal of Autism Developmental Disorder, 44, 1897-1907.

Lei 11.794, de 8 de outubro de 2008. (2008). Regulamenta o inciso VII do $\S 1$ o do art. 225 da Constituição Federal, estabelecendo procedimentos para o uso científico de animais; revoga a Lei $n$ o 6.638, de 8 de maio de 1979; e dá outras providências. Brasília, 2008. Recuperado em 06 novembro, 2014, de http://www. planalto.gov.br/ccivil_03/_ato20072010/2008/lei/l11794.htm

Leitão, L. G. (2004). Relações terapêuticas: um estudo exploratório sobre equitação psico-educacional (EPE) e autismo. Análise Psicológica, 22(2), 335-354.

Leitão, L. G. (2008). Sobre a equitação terapêutica: uma abordagem crítica, Análise Psicológica, 26(1), 81-100.

Levinson, B. M. (1965). Pet psychotherapy: use of household pets in the treatment of behavior disorder in childhood. Psychological Reports, 17, 695-698. Recuperado em 27 janeiro, 2017, de http://journals. sagepub.com/doi/pdf/10.2466/pr0.1965.17.3.69 5

Memisevikj, X., \& Hodzhikj, S. (2010). The effects of equine-assisted therapy in improving the psycho-social functioning of children with autismo. Journal of Special Education and Rehabilitation, 11(3-4), 57-67. 
Minerbo, M. (2002). C(ã)ES-terapeutas: o enquadre a serviço do método na análise de uma adolescente. Estilos da Clínica, $7(12), 12-27$.

Muszkat, M. (2005). Desenvolvimento e Neuroplasticidade. In C. B. Mello, M. C. Miranda, \& M. Muszkat (Eds.), NeuroPsicologia do Desenvolvimento - conceitos e abordagens (pp. 26-45). São Paulo: Mennon.

Ory, M. G. \& Goldberg, E. L. (1983). Pet possession and well-being in elderly women, RESEARCH ON AGING, 5(3), 389-409. Recuperado em 27 janeiro, 2017, de http://journals. sagepub.com/doi/pdf/10.1177/0164027583005 003007

Paula, C., Duarte, C., \& Bordini, I. (2007). Prevalence of mental health problems in children and adolescents from the outskirts of Sao Paulo City: treatment needs and service capacity evaluation. Revista Brasileira de Psiquiatria, 29(1), 11-17. Recuperado em 27 janeiro, 2017, de http://dx.doi.org/10.1590/S1516-44462006005000012

Pereira, M. J. F., Pereira, L., \& Ferreira, M. L. (2007). Os benefícios da Terapia Assistida por Animais: uma revisão bibliográfica, Saúde Coletiva, 4(14), 62-66. Recuperado em 08 outubro, 2014, de http://www. redalyc.org/articulo.oa?id=84201407

Poresky, R. H. (1997). The companion animal bonding scale: Internal consistency and factor structure when administered by telephone, Psychological Reports, 80, 937-939. Recuperado em 27 janeiro, 2017, de http://journals. sagepub.com/doi/pdf/10.2466/pr0.1997.80.3.93 7

Poutechi, R. C., \& Coutinho, M. J. (2011). A intervenção da terapia assistida por animais nas atividades de vida diária de crianças com paralisia cerebral, Anuário da produção de iniciação científica discente, 14(28), 77-86.

Rose, P., Cannas, E., \& Cantiello, P. R. (2011). Donkey-assisted rehabilitation program for children: a pilot study, Annali dell'Ist. Super. Sanità, 47(4), 391-396. Recuperado em 26 março, 2015, http://www.scielosp.org/scielo.php?script=sci_arttext\&nrm=iso \&lng $=$ pt\&tIng $=$ pt\&pid $=$ S0021-25712011000400011

Salgueiro, E., Nunes, L., Barros, A., Maroco, J., Salgueir, A. I., \& Santos, M. E. (2012). Effects of a dolphin interaction program on children with autism spectrum disorders - an exploratory research, BioMed Central Research Notes, 5, 1-8.

Sobo, E. J., Eng, B., \& Kassity-Krich, N. (2006). Canine visitation (pet) therapy: Pilot data on decreases in child pain perception, Journal of Holistic Nursing, 24(1), 51-57. Recuperado em 27 janeiro, 2017, de 
http://jmfarns.tripod.com/sitebuildercontent/sitebuilderfiles/cvt .pdf

Vaccari, A. M. H., \& Almeida, F. A. (2007,). A importância da visita de animais de estimação na recuperação de crianças hospitalizadas, Einstein, 5(2), 111-116. Recuperado em 30 abril, 2015,

de

http://apps. einstein. br/revista/arquivos/PDF/419-Einstein52_Online_AO419_pg111-116.pdf

Vivaldini, V. H. (2011). Terapia assistida por animais: uma abordagem lúdica em reabilitação clínica de pessoas com deficiência intelectual. (Dissertação de mestrado não publicada). Universidade Metodista de São Paulo, São Bernardo do Campo, SP, Brasil.

Ylvisaker, M., Adelson, D., Braga, L. W., Burnett, S. M., Glang, A., Feeney, T., ... Todis, B. (2005). Rehabilitation and ongoing support after pediatric TBI: twenty years of progress, J Head Trauma Rehabil, 20(1), 95-109.

\section{Endereço para correspondência \\ Jéssica Riedi Souza Marinho \\ Centro Universitário FADERGS - Laureate Universities \\ Rua Francisca Lechner, 113, Rubem Berta, CEP 91180-560, Porto Alegre - RS, Brasil \\ Endereço eletrônico: jessica.riedism@gmail.com}

\section{Renata de Souza Zamo}

Centro Universitário FADERGS - Laureate Universities

Rua Caju, 28 conjunto 503, Petrópolis, CEP 90690-310, Porto Alegre - RS, Brasil

Endereço eletrônico: psicozamo@hotmail.com

Recebido em: 01/08/2015

Reformulado em: 27/01/2017

Aceito em: 03/03/2017

\section{Notas}

* Psicóloga, egressa do Centro Universitário FADERGS - Laureate Universities.

** Psicóloga, Professora do Centro Universitário FADERGS - Laureate Universities, Mestre e Doutora em Psicologia do Programa de Pós-Graduação em Psicologia da Universidade Federal do Rio Grande do Sul (UFRGS).

Este artigo de revista Estudos e Pesquisas em Psicologia é licenciado sob uma Licença Creative Commons Atribuição-Não Comercial 3.0 Não Adaptada. 